\title{
Comparison on thrust characteristic of linear oscillatory actuators
}

\begin{abstract}
This paper is aimed to analyze the thrust characteristics for two types of Linear Oscillatory Actuator using 3D-Finite Element Method (FEM). The coil currents are analyzed according to the variation displacement of moving yoke of the first one of the LOA. The thrust, normal force and cogging force are analyzed according to the variation of the airgap length, thickness taper and high taper of second LOA. The size of both model of LOA is same but comparison refers to the characteristic of voltage, coil current, number of winding and force. The result of this project can be see from the graph of characteristic both model. Finally, the best characteristics of LOA can be constructed from the result.
\end{abstract}

Keyword: Finite element analysis; Linear oscillatory motor; Taper gap; Thrust characteristics 